\title{
Strong convergence of hybrid Bregman projection algorithm for split feasibility and fixed point problems in Banach spaces
}

\author{
Jin-Zuo Chen, Hui-Ying Hu, Lu-Chuan Ceng* \\ Department of Mathematics, Shanghai Normal University, Shanghai, 200234, China. \\ Communicated by $\mathrm{X}$. Qin
}

\begin{abstract}
In this paper, we consider and study split feasibility and fixed point problems involved in Bregman quasi-strictly pseudocontractive mapping in Banach spaces. It is proven that the sequences generated by the proposed iterative algorithm converge strongly to the common solution of split feasibility and fixed point problems. (c) 2017 All rights reserved.
\end{abstract}

Keywords: Split feasibility problem, fixed point problem, Bregman quasi-strictly pseudo-contractive mapping, Bregman projection, strong convergence.

2010 MSC: 47J25, 47H10, 65J15, 90C25.

\section{Introduction}

Throughout this paper, let $E_{1}$ and $E_{2}$ be two p-uniformly convex real Banach spaces which are also uniformly smooth. Let $C$ and $Q$ be nonempty closed convex sets of $E_{1}$ and $E_{2}$, respectively. Let $A$ be a bounded linear operator from $E_{1}$ to $E_{2}$ with its adjoint $A^{*}$. Let $T$ be a nonlinear mapping from $C$ to itself. We use Fix $(T)$ to denote the set of all fixed points of the mapping $T$, that is, $F i x(T)=\{x \in C: T x=x\}$.

This paper is concerned with studying the following split feasibility and fixed point problems:

$$
\text { Find } x^{*} \in \mathrm{C} \cap \operatorname{Fix}(\mathrm{T}) \text { such that } A x^{*} \in \mathrm{Q} \text {. }
$$

Let $\Gamma=\left\{x^{*}: x^{*} \in C \cap \operatorname{Fix}(T)\right.$ such that $\left.A x^{*} \in Q\right\}$ be the set of all solutions of (1.1). In the sequel, we assume $\Gamma \neq \emptyset$. A special case of (1.1) is the following split feasibility problem (in short, SFP):

$$
\text { Find } x^{*} \in \mathrm{C} \text { such that } A x^{*} \in \mathrm{Q} \text {. }
$$

Let $\Gamma_{0}=\left\{x^{*}: x^{*} \in \mathrm{C}\right.$ such that $\left.A x^{*} \in \mathrm{Q}\right\}$ be the set of all solutions of (1.2). Then, we have that $\Gamma_{0}$ is a closed and convex subset of $E_{1}$.

\footnotetext{
*Corresponding author

Email addresses: chanjanegeoger@163.com (Jin-Zuo Chen), huiying1117@hotmail .com (Hui-Ying Hu), zenglc@hotmail .com (Lu-Chuan Ceng)
}

doi:10.22436/jnsa.010.01.19 
The theory of the SFP was first introduced and learned by Censor and Elfving [13] in finite-dimensional space for modeling inverse problems which arise from phase retrievals and in image reconstruction has been discussed in the last two decades and much intensively in the last ten years. A large number of algorithms related to the SFP have been studied, see, for example $[5,6,14]$ and the references therein. Recently, it has been found that the SFP can be used in intensity modulated radiation therapy, please see $[12,14,15]$ and the references therein. The algorithm suggested by Censor and Elfving in [13] involves the computation of the inverse $A^{-1}$, so it can not be widely used. A seemingly more popular algorithm is the CQ algorithm [5]:

$$
x_{n+1}=P_{C}\left(I-\gamma A^{*}\left(I-P_{Q}\right) A\right) x_{n}, \quad n \geqslant 0,
$$

where $x_{0} \in \mathcal{H}_{1}$ (a Hilbert space) and $\gamma \in\left(0, \frac{2}{\lambda}\right)$, with $\lambda$ being the largest eigenvalue of the matrix $A^{*} A$. Recently, the SFP was studied in a more general framework, for example, Banach spaces. More specifically, Schöpfer et al. [21] proposed the following algorithm in p-uniformly convex and uniformly smooth real Banach spaces:

$$
x_{n+1}=\Pi_{C} J^{*}\left(J x_{n}-\gamma A^{*} J\left(I-P_{Q}\right) A x_{n}\right),
$$

where $\Pi_{C}$ denotes the Bregman projection and $J$ the duality mapping, they established weak convergence of algorithm (1.4) under some mild conditions. Obviously the above algorithm (1.4) covers the CQ algorithm (1.3) as a special case.

It is worth pointing out that only weak convergence result is established in [21]. However, the strong convergence is more acceptable than the weak convergence in some practical applications. Wang [24] considered the following iterative algorithm for multiple-sets split feasibility problem in p-uniformly convex and uniformly smooth real Banach spaces:

$$
\left\{\begin{array}{l}
y_{n}=T_{n} x_{n}, \\
D_{n}=\left\{v \in E_{1}: \Delta_{p}\left(y_{n}, v\right) \leqslant \Delta_{p}\left(x_{n}, v\right)\right\} \\
E_{n}=\left\{v \in E_{1}:\left\langle x_{n}-v, J_{p} \bar{x}-J_{p} x_{n}\right\rangle \geqslant 0\right\} \\
x_{n+1}=\Pi_{D_{n} \cap E_{n}} \bar{x} .
\end{array}\right.
$$

Using the idea in the work of Nakajo and Takahashi [18], Wang proved the strong convergence of iterative algorithm (1.5). Subsequently, Takahashi [22] proposed the following hybrid projection algorithm for the SFP in uniformly convex and uniformly smooth real Banach spaces:

$$
\left\{\begin{array}{l}
z_{n}=x_{n}-r J_{E_{1}}^{-1} A^{*} J_{E_{2}}\left(A x_{n}-P_{Q} A x_{n}\right), \\
C_{n}=\left\{v \in E_{1}:\left\langle z_{n}-v, J_{E_{1}}\left(x_{n}-z_{n}\right)\right\rangle \geqslant 0\right\}, \\
Q_{n}=\left\{v \in E_{1}:\left\langle x_{n}-v, J_{E_{1}}\left(x_{1}-x_{n}\right)\right\rangle \geqslant 0\right\}, \\
x_{n+1}=P_{C_{n} \cap Q_{n}} x_{1} .
\end{array}\right.
$$

Basing mainly on the hybrid method, he proved the strong convergence of iterative algorithm (1.6).

On the other hand, in 1967, Bregman [4] used the so-called Bregman distance function to design and analyze feasibility and optimization algorithms. After that, many authors found that the so-called Bregman distance function could be applied in different ways in order to construct iterative algorithms for solving not only feasibility and optimization problems, but also variational inequality problems, equilibria problems, fixed points problems and so on (see, e.g., $[1,3,7-11,16,17,19,20,26-28]$ and the references therein). The fixed point theory with respect to Bregman distance has been studied in the last decade and a lot of good results were published intensively in the last five years. Many authors concentrated their energies on constructing the fixed point of Bregman nonlinear operators by utilizing the Bregman distance and the Bregman projection, see [23, 25] and the references therein. In 2015, Wang [25] studied a new hybrid Bregman projection iterative algorithm for Bregman quasi-strictly pseudo-contractive mapping and proved strong convergence result in reflexive Banach spaces. In particularly, he proposed the following 
iterative method:

$$
\left\{\begin{array}{l}
x_{0} \in C \text { chosen arbitrarily, } \\
C_{1}=C \\
x_{1}=P_{C_{1}} x_{0} \\
C_{n+1}=\left\{v \in C_{n}: D_{f}\left(x_{n}, T x_{n}\right) \leqslant \frac{1}{1-k}\left\langle\nabla f\left(x_{n}\right)-\nabla f\left(T x_{n}\right), x_{n}-v\right\rangle\right\} \\
x_{n+1}=P_{C_{n+1}} x_{0},
\end{array}\right.
$$

where $k \in[0,1)$. Then the sequence $\left\{x_{n}\right\}$ converges strongly to $p=P_{F i x(T)} x_{0}, P_{F i x(T)}$ is the Bregman projection of $\mathrm{E}$ onto Fix $(\mathrm{T})$.

In this paper, motivated and inspired by the above research work going on in this field, we propose a new hybrid projection method for solving split feasibility and fixed point problems (1.1) involved in Bregman quasi-strictly pseudo-contractive mapping in p-uniformly convex and uniformly smooth real Banach spaces. Our modification is mainly based on the schemes (1.5), (1.6) and (1.7). Furthermore, we will prove the strong convergence theorem for the proposed algorithm.

\section{Preliminaries}

Let $1<q \leqslant 2 \leqslant p$ with $1 / p+1 / q=1$. Let $E$ be a real Banach space.

The modulus of convexity of $E$ is the function $\delta_{E}:(0,2] \rightarrow[0,1]$ defined by

$$
\delta_{E}(\epsilon)=\inf \left\{1-\frac{\|x+y\|}{2}: x, y \in S(E),\|x-y\| \geqslant \epsilon\right\},
$$

for any $x, y$ on the unit sphere $S(E)=\{x \in E:\|x\|=1\}$. E is called uniformly convex, if $\delta_{E}(\epsilon)>0$ for any $\epsilon \in(0,2] ; p$-uniformly convex, if there exists $c_{p}>0$ such that $\delta_{E}(\epsilon) \geqslant c_{p} \epsilon^{p}$ for any $\epsilon \in(0,2]$.

The modulus of smoothness of $E$ is the function $\rho_{E}:[0, \infty) \rightarrow[0, \infty)$ defined by

$$
\rho_{\mathrm{E}}(\mathrm{t})=\sup \left\{\frac{1}{2}(\|x+y\|-\|x-y\|)-1: x \in S(E),\|y\|=t\right\} .
$$

$E$ is called uniformly smooth, if $\lim _{t \rightarrow 0} \rho_{E}(t) / t=0$. By setting $1<q \leqslant 2 \leqslant p$, a Banach space $E$ is called q-uniformly smooth, if there exists $C_{q}>0$ such that $\rho_{E}(t) \leqslant C_{q} t^{q}$ for all $t>0$. We assume that $E$ is p-uniformly convex and uniformly smooth, which implies that its dual space, $E^{*}$, is q-uniformly smooth and uniformly convex. In this situation, it is known that the duality mapping $J_{\mathrm{E}}^{\mathrm{p}}$ is one-to-one, single-valued and satisfies $\mathrm{J}_{\mathrm{E}}^{\mathrm{p}}=\left(\mathrm{J}_{\mathrm{E}^{*}}^{\mathrm{q}}\right)^{-1}$, where $\mathrm{J}_{\mathrm{E}^{*}}^{\mathrm{q}}$ is the duality mapping of $\mathrm{E}^{*}$.

The q-uniformly smooth spaces have the following conclusion.

Lemma 2.1 ([24]). If $\mathrm{E}$ is a $\mathrm{q}$-uniformly smooth space, then there is a constant $\mathrm{C}_{\mathrm{q}}>0$ such that

$$
\|x-y\|^{q} \leqslant\|x\|^{q}-q\left\langle y, J_{E}^{q}(x)\right\rangle+C_{q}\|y\|^{q},
$$

for all $\mathrm{x}, \mathrm{y} \in \mathrm{E}$, where $\mathrm{C}_{\mathrm{q}}>0$ is the $\mathrm{q}$-uniformly smoothness constant of $\mathrm{E}$ and $\mathrm{J}_{\mathrm{E}}^{\mathrm{q}}$ is the duality mapping from $\mathrm{E}$ into $2^{\mathrm{E}^{*}}$ defined by

$$
\mathrm{J}_{\mathrm{E}}^{\mathrm{q}}(\mathrm{x})=\left\{x^{*} \in \mathrm{E}^{*}:\left\langle x, x^{*}\right\rangle=\|x\|^{\mathrm{q}},\left\|x^{*}\right\|=\|x\|^{\mathrm{q}-1}\right\}, \quad \forall x, y \in \mathrm{E} .
$$

Given a Gâteaux differentiable convex function $f: E \rightarrow \mathbb{R}$, the Bregman distance with respect to $f$ is defined by

$$
\Delta_{f}(x, y)=f(y)-f(x)-\left\langle f^{\prime}(x), y-x\right\rangle, \quad \forall x, y \in E .
$$


It is noteworthy that the duality mapping $J_{p}$ is the derivative of the function $f_{p}=\frac{1}{p}\|x\|^{p}$. Then the Bregman distance with respect to $f_{p}$ is given by

$$
\begin{aligned}
\Delta_{p}(x, y) & =\frac{1}{q}\|x\|^{p}-\left\langle J_{E}^{p} x, y\right\rangle+\frac{1}{p}\|y\|^{p} \\
& =\frac{1}{p}\left(\|y\|^{p}-\|x\|^{p}\right)+\left\langle J_{E}^{p} x, x-y\right\rangle \\
& =\frac{1}{q}\left(\|x\|^{p}-\|y\|^{p}\right)-\left\langle J_{E}^{p} x-J_{E}^{p} y, x\right\rangle .
\end{aligned}
$$

From the definition of $\Delta_{\mathfrak{p}}(\cdot, \cdot)$, we get

$$
\Delta_{\mathrm{p}}(x, y)=\Delta_{\mathfrak{p}}(x, z)+\Delta_{\mathfrak{p}}(z, y)+\left\langle z-y, J_{E}^{p} x-J_{E}^{p} z\right\rangle,
$$

and

$$
\Delta_{p}(x, y)+\Delta_{p}(y, x)=\left\langle x-y, J_{E}^{p} x-J_{E}^{p} y\right\rangle,
$$

for any $x, y, z \in E$. All in all, the Bregman distance is not a metric because of the lack of symmetry. For the p-uniformly convex space, the metric and Bregman distance has the following relation

$$
\tau\|x-y\|^{p} \leqslant \Delta_{p}(x, y) \leqslant\left\langle x-y, J_{E}^{p} x-J_{E}^{p} y\right\rangle,
$$

where $\tau>0$. Obviously, if $\left\{x_{n}\right\}$ and $\left\{y_{n}\right\}$ are both bounded sequences of a p-uniformly convex and uniformly smooth space $E$, then $x_{n}-y_{n} \rightarrow 0$ as $n \rightarrow \infty$ implies that $\Delta_{p}\left(x_{n}, y_{n}\right) \rightarrow 0$ as $n \rightarrow \infty$.

Projections are an important tool for our work in this paper. We can define metric projection $\mathrm{P}_{C}$ as follows

$$
P_{C} x=\operatorname{argmin}_{y \in C}\|x-y\|, \quad x \in E .
$$

Metric projection $\mathrm{P}_{\mathrm{C}}$ can be characterized by the following variational inequality

$$
\left\langle J_{\mathrm{E}}^{\mathrm{p}}\left(x-\mathrm{P}_{\mathrm{C}} x\right), z-\mathrm{P}_{\mathrm{C}} x\right\rangle \leqslant 0, \quad z \in \mathrm{C} .
$$

Likewise, one can define the Bregman projection

$$
\Pi_{C} x=\operatorname{argmin}_{y \in C} \Delta_{p}(x, y), \quad x \in E,
$$

is the unique minimizer of the Bregman distance. The Bregman projection can also be characterized by the following variational inequality

$$
\left\langle\mathrm{J}_{\mathrm{E}}^{\mathrm{p}} x-\mathrm{J}_{\mathrm{E}}^{\mathrm{p}} \Pi_{\mathrm{C}} x, z-\Pi_{\mathrm{C}} x\right\rangle \leqslant 0, \quad z \in \mathrm{C},
$$

from which one has

$$
\Delta_{\mathfrak{p}}\left(\Pi_{\mathrm{C}} x, z\right) \leqslant \Delta_{\mathfrak{p}}(x, z)-\Delta_{\mathfrak{p}}\left(x, \Pi_{\mathrm{C}} x\right), \quad z \in \mathrm{C} .
$$

The metric projection and the Bregman projection with respect to $f_{2}$ are coincident in a Hilbert space, but in a more general framework, they are totally different. What is important is that the metric projection can not share property (2.5) as the Bregman projection in Banach spaces.

Following [2], we study the function $V_{p}: E^{*} \times E \rightarrow[0, \infty)$ associated with $f_{p}$, which is defined by

$$
V_{p}(\bar{x}, x)=\frac{1}{q}\|\bar{x}\|^{q}-\langle\bar{x}, x\rangle+\frac{1}{p}\|x\|^{p}, \quad x \in E, \bar{x} \in E^{*} .
$$

Then $V_{p}$ is nonnegative and

$$
\mathrm{V}_{\mathrm{p}}(\bar{x}, x)=\Delta_{\mathrm{p}}\left(\mathrm{J}_{\mathrm{E}}^{\mathrm{q}} \overline{\mathrm{x}}, \mathrm{x}\right), \quad x \in \mathrm{E}, \overline{\mathrm{x}} \in \mathrm{E}^{*}
$$


Moreover, by the subdifferential inequality, we have

$$
V_{p}(\bar{x}, x)+\left\langle\bar{y}, J_{E^{*}}^{q} \bar{x}-x\right\rangle \leqslant V_{p}(\bar{x}+\bar{y}, x), \quad x \in E, \bar{x}, \bar{y} \in E^{*} .
$$

In addition, $\mathrm{V}_{\mathrm{p}}$ is convex in the first variable. Thus, for all $z \in \mathrm{E}$,

$$
\Delta_{p}\left(J_{E^{*}}^{q}\left(\sum_{i=1}^{N} t_{i} J_{E}^{p}\left(x_{i}\right)\right), z\right) \leqslant \sum_{i=1}^{N} t_{i} \Delta_{p}\left(x_{i}, z\right),
$$

where $\left\{x_{i}\right\}_{i=1}^{N} \subset E$ and $\left\{t_{i}\right\}_{i=1}^{N} \subset(0,1)$ with $\sum_{i=1}^{N} t_{i}=1$. For more details about $V_{p}$, please see [2].

Very recently, Ugwunnadi et al. [23] introduced the concept of Bregman quasi-strictly pseudocontractive mapping and proved the strong convergence by using hybrid Bregman projection iterative algorithm for a Bregman quasi-strictly pseudo-contractive mapping.

Definition 2.2. A mapping $\mathrm{T}: \mathrm{C} \rightarrow \mathrm{C}$ is said to be Bregman quasi-strictly pseudo-contractive mapping, if there exists a constant $k \in[0,1)$ and $\operatorname{Fix}(T) \neq \emptyset$ such that

$$
\Delta_{\mathfrak{p}}\left(T x, x^{*}\right) \leqslant \Delta_{\mathfrak{p}}\left(x, x^{*}\right)+k \Delta_{p}(T x, x), \quad \forall x \in C, x^{*} \in \operatorname{Fix}(T) .
$$

Definition 2.3. A mapping $T: C \rightarrow C$ is said to be Bregman quasi-nonexpansive mapping, if $F i x(T) \neq \emptyset$ such that

$$
\Delta_{\mathrm{p}}\left(\mathrm{T} x, \mathrm{x}^{*}\right) \leqslant \Delta_{\mathfrak{p}}\left(x, x^{*}\right), \quad \forall x \in \mathrm{C}, x^{*} \in \operatorname{Fix}(\mathrm{T}) .
$$

Definition 2.4. A mapping $T: C \rightarrow C$ is said to be closed, if for any sequence $\left\{x_{n}\right\} \subset C$ with $x_{n} \rightarrow x \in C$ and $T x_{n} \rightarrow y \in C$ as $n \rightarrow \infty$, then $T x=y$.

We shall adopt the notation $x_{n} \rightarrow x$ means that $\left\{x_{n}\right\}$ converges strongly to $x$. Now, we give some examples of a Bregman quasi-strictly pseudo-contractive mapping.

Example 2.5 ([25]). Let $E$ be a smooth space, and define $f(x)=\|x\|^{2}$ for all $x \in E$. Let $x_{0} \neq 0$ be any element of $E, T: E \rightarrow E$ be defined as follows:

$$
T(x)= \begin{cases}\left(1 / 2+1 / 2^{n+1}\right) x_{0}, & x=\left(1 / 2+1 / 2^{n}\right) x_{0}, \\ -x, & x \neq\left(1 / 2+1 / 2^{n}\right) x_{0},\end{cases}
$$

for all $n \geqslant 1$. Then $T$ is a Bregman quasi-strictly pseudo-contractive mapping.

Example 2.6 ([23]). Let $E=\mathbb{R}$ and define $T, f:[-1,0] \rightarrow \mathbb{R}$ by $f(x)=x$ and $T(x)=2 x$ for all $x \in[-1,0]$. Then $T$ is a Bregman quasi-strictly pseudo-contractive mapping.

\section{Main results}

In this section, we introduce the following algorithm and prove strong convergence theorem for finding the common solution of split feasibility and fixed point problems.

Theorem 3.1. Let $\mathrm{E}_{1}$ and $\mathrm{E}_{2}$ be two p-uniformly convex real Banach spaces which are also uniformly smooth. Let $C$ and $Q$ be nonempty closed convex sets of $E_{1}$ and $E_{2}$, respectively. Let $A: E_{1} \rightarrow E_{2}$ be a bounded linear operator with its adjoint $\mathrm{A}^{*}: \mathrm{E}_{2}^{*} \rightarrow \mathrm{E}_{1}^{*}$. Let $\mathrm{T}$ be a closed Bregman quasi-strictly pseudo-contractive mapping from $\mathrm{C}$ to itself. Let the sequence $\left\{x_{n}\right\}$ be iteratively generated by $x_{1}=x_{0} \in C, D_{1}=C_{1}=C$,

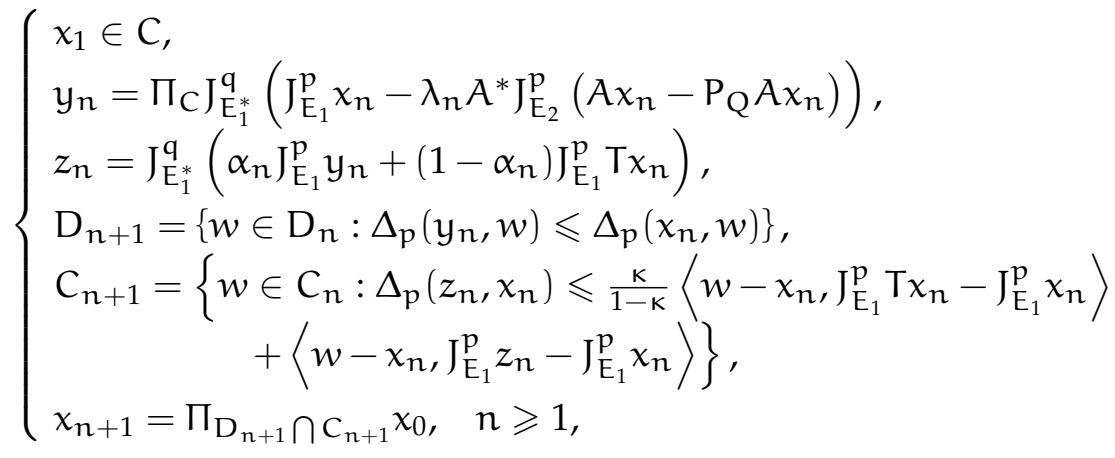


where $\mathrm{k} \in[0,1)$. Assume that $\left\{\alpha_{\mathrm{n}}\right\} \subset[\mathrm{c}, \mathrm{d}] \subset(0,1)$ and $\left\{\lambda_{\mathrm{n}}\right\} \subset[\mathrm{a}, \mathrm{b}] \subset\left(0,\left(\frac{\mathrm{q}}{\mathrm{C}_{\mathrm{q}}\|\mathrm{A}\| \mathrm{q}}\right)^{\frac{1}{\mathrm{q}-1}}\right)$. Then the sequence $\left\{x_{n}\right\}$ defined by (3.1) converges strongly to $\Pi_{\Gamma} x_{0}$.

Proof. By taking $x^{*} \in \Gamma, x^{\prime} \in \mathrm{C}$, by the definition of $\mathrm{T}$, we have

$$
\Delta_{\mathfrak{p}}\left(T x^{\prime}, x^{*}\right) \leqslant \Delta_{\mathfrak{p}}\left(x^{\prime}, x^{*}\right)+\kappa \Delta_{p}\left(T x^{\prime}, x^{\prime}\right) .
$$

From (2.1), we get

$$
\Delta_{\mathfrak{p}}\left(\mathrm{T} x^{\prime}, x^{*}\right)=\Delta_{\mathfrak{p}}\left(\mathrm{T} x^{\prime}, x^{\prime}\right)+\Delta_{\mathfrak{p}}\left(x^{\prime}, x^{*}\right)+\left\langle x^{\prime}-x^{*}, \mathrm{~J}_{\mathrm{E}_{1}}^{\mathrm{p}} \mathrm{T} x^{\prime}-\mathrm{J}_{\mathrm{E}_{1}}^{\mathrm{p}} x^{\prime}\right\rangle,
$$

which implies that

$$
\Delta_{\mathrm{p}}\left(\mathrm{T} x^{\prime}, x^{\prime}\right) \leqslant \frac{1}{1-\kappa}\left\langle x^{*}-x^{\prime}, \mathrm{J}_{\mathrm{E}_{1}}^{\mathrm{p}} \mathrm{T} x^{\prime}-\mathrm{J}_{\mathrm{E}_{1}}^{\mathrm{p}} x^{\prime}\right\rangle .
$$

Let $\left\{x_{n}\right\}$ be a sequence in Fix $(T)$ such that $x_{n} \rightarrow z$ as $n \rightarrow \infty$. From (3.2), we obtain

$$
\Delta_{\mathfrak{p}}(\mathrm{T} z, z) \leqslant \frac{1}{1-\mathrm{K}}\left\langle x_{\mathrm{n}}-z, \mathrm{~J}_{\mathrm{E}_{1}}^{\mathrm{p}} \mathrm{T} z-\mathrm{J}_{\mathrm{E}_{1}}^{\mathrm{p}} z\right\rangle .
$$

By setting $n \rightarrow \infty$ in the above inequality, we have $\Delta_{\mathfrak{p}}(\mathrm{T} z, z) \leqslant 0$, it follows from (2.2) that $\mathrm{T} z=z$. Therefore, $\operatorname{Fix}(\mathrm{T})$ is closed. tively,

Next, let $z_{1}, z_{2} \in \operatorname{Fix}(T)$, for given $t \in(0,1)$ and put $z=t z_{1}+(1-t) z_{2}$. From (3.2), we obtain respec-

$$
\Delta_{\mathrm{p}}(\mathrm{T} z, z) \leqslant \frac{1}{1-\mathrm{K}}\left\langle z_{1}-z, \mathrm{~J}_{\mathrm{E}_{1}}^{\mathrm{p}} \mathrm{T} z-\mathrm{J}_{\mathrm{E}_{1}}^{\mathrm{p}} z\right\rangle,
$$

and

$$
\Delta_{\mathrm{p}}(\mathrm{T} z, z) \leqslant \frac{1}{1-\mathrm{K}}\left\langle z_{2}-z, \mathrm{~J}_{\mathrm{E}_{1}}^{\mathrm{p}} \mathrm{T} z-\mathrm{J}_{\mathrm{E}_{1}}^{\mathrm{p}} z\right\rangle .
$$

By multiplying (3.3) by $t$ and (3.4) by $1-t$, we have

$$
\Delta_{\mathrm{p}}(\mathrm{T} z, z) \leqslant \frac{1}{1-\mathrm{\kappa}}\left\langle z-z, \mathrm{~J}_{\mathrm{E}_{1}}^{\mathrm{p}} \mathrm{T} z-\mathrm{J}_{\mathrm{E}_{1}}^{\mathrm{p}} z\right\rangle,
$$

and by setting $n \rightarrow \infty$ in the above inequality, we have $\Delta_{p}(T z, z) \leqslant 0$. It follows from (2.2) that $T z=z$. Therefore, $\operatorname{Fix}(T)$ is convex. Since $\Gamma_{0}$ is a closed convex subset of $E_{1}$, we obtain that $\Gamma$ is closed convex.

Now from (3.1), we know $D_{n}$ is closed for each $n \geqslant 1$. Note that $\Delta_{p}\left(y_{n}, w\right) \leqslant \Delta_{p}\left(x_{n}, w\right)$ is equivalent to

$$
\left\langle\mathrm{J}_{\mathrm{E}_{1}}^{\mathrm{p}} \mathrm{x}_{\mathrm{n}}-\mathrm{J}_{\mathrm{E}_{1}}^{\mathrm{p}} \mathrm{y}_{\mathrm{n}}, w\right\rangle \leqslant \frac{1}{\mathrm{q}}\left(\left\|\mathrm{x}_{\mathrm{n}}\right\|^{\mathrm{p}}-\left\|\mathrm{y}_{\mathrm{n}}\right\|^{\mathrm{p}}\right),
$$

so that $D_{n}$ is a halfspace. Therefore, we get $D_{n}$ is convex immediately.

For $n=1, C_{1}=C$ is closed convex essentially. Assume that $C_{n}$ is closed convex for $n>1$. For $w \in \mathrm{C}_{\mathrm{n}+1}$, we obtain

$$
\Delta_{\mathrm{p}}\left(z_{n}, x_{n}\right) \leqslant \frac{\kappa}{1-\kappa}\left\langle w-x_{n}, J_{E_{1}}^{p} \mathrm{~T}_{n}-J_{\mathrm{E}_{1}}^{\mathrm{p}} x_{n}\right\rangle+\left\langle w-x_{n}, \mathrm{~J}_{\mathrm{E}_{1}}^{\mathrm{p}} z_{n}-\mathrm{J}_{\mathrm{E}_{1}}^{\mathrm{p}} x_{n}\right\rangle,
$$

since $\left\langle\cdot, \mathrm{J}_{\mathrm{E}_{1}}^{\mathrm{p}} \mathrm{T} x_{\mathrm{n}}-\mathrm{J}_{\mathrm{E}_{1}}^{\mathrm{p}} \mathrm{x}_{\mathrm{n}}\right\rangle$ and $\left\langle\cdot, \mathrm{J}_{\mathrm{E}_{1}}^{\mathrm{p}} z_{\mathrm{n}}-\mathrm{J}_{\mathrm{E}_{1}}^{\mathrm{p}} \mathrm{x}_{\mathrm{n}}\right\rangle$ are continuous and linear in $\mathrm{E}_{1}$, we get $\mathrm{C}_{\mathrm{n}}$ is closed convex.

Let $x^{*} \in \Gamma$ and let $v_{n}=A x_{n}-P_{Q} A x_{n}$. It follows from (2.3) that

$$
\begin{aligned}
\left\langle J_{\mathrm{E}_{2}}^{\mathrm{p}} \nu_{n}, A x_{n}-A x^{*}\right\rangle & =\left\|A x_{n}-P_{Q} A x_{n}\right\|^{p}+\left\langle J_{E_{2}}^{p} \nu_{n}, P_{Q} A x_{n}-A x^{*}\right\rangle \\
& \geqslant\left\|A x_{n}-P_{Q} A x_{n}\right\|^{p} .
\end{aligned}
$$


By applying Lemma 2.1, we have

$$
\begin{aligned}
& \Delta_{\mathrm{p}}\left(\mathrm{y}_{\mathrm{n}}, x^{*}\right) \leqslant \Delta_{\mathrm{p}}\left(\mathrm{J}_{\mathrm{E}_{1}^{*}}^{\mathrm{q}}\left(\mathrm{J}_{\mathrm{E}_{1}}^{\mathrm{p}} x_{\mathrm{n}}-\lambda_{n} A^{*} \mathrm{~J}_{\mathrm{E}_{2}}^{\mathrm{p}} v_{\mathrm{n}}\right), x^{*}\right) \\
& =\frac{1}{\mathrm{q}}\left\|\mathrm{J}_{\mathrm{E}_{1}}^{\mathrm{p}} x_{n}-\lambda_{n} A^{*} \mathrm{~J}_{\mathrm{E}_{2}}^{\mathrm{p}} \nu_{n}\right\|^{\mathrm{q}}-\left\langle\mathrm{J}_{\mathrm{E}_{1}}^{\mathrm{p}} x_{n}, x^{*}\right\rangle+\lambda_{n}\left\langle\mathrm{~J}_{\mathrm{E}_{2}}^{\mathrm{p}} \nu_{n}, A x^{*}\right\rangle+\frac{1}{\mathrm{p}}\left\|x^{*}\right\|^{\mathrm{p}} \\
& \leqslant \frac{1}{\mathrm{q}}\left\|\mathrm{J}_{\mathrm{E}_{1}}^{\mathrm{p}} x_{n}\right\|^{\mathrm{q}}-\lambda_{n}\left\langle A x_{n}, J_{\mathrm{E}_{2}}^{\mathrm{p}} \nu_{n}\right\rangle+\frac{\mathrm{C}_{\mathrm{q}}\left(\lambda_{n}\|A\|\right)^{\mathrm{q}}}{\mathrm{q}}\left\|\mathrm{J}_{\mathrm{E}_{2}}^{\mathrm{p}} \nu_{n}\right\|^{\mathrm{q}} \\
& -\left\langle J_{\mathrm{E}_{1}}^{\mathrm{p}} x_{n}, x^{*}\right\rangle+\lambda_{n}\left\langle J_{\mathrm{E}_{2}}^{\mathrm{p}} v_{n}, A x^{*}\right\rangle+\frac{1}{\mathrm{p}}\left\|x^{*}\right\|^{\mathrm{p}} \\
& =\frac{1}{q}\left\|x_{n}\right\|^{p}-\left\langle J_{E_{1}}^{p} x_{n}, x^{*}\right\rangle+\frac{1}{p}\left\|x^{*}\right\|^{p}+\lambda_{n}\left\langle J_{E_{2}}^{p} v_{n}, A x^{*}-A x_{n}\right\rangle \\
& +\frac{C_{q}\left(\lambda_{n}\|A\|\right)^{q}}{q}\left\|J_{E_{2}}^{p} v_{n}\right\|^{q} \\
& =\Delta_{\mathrm{p}}\left(x_{n}, x^{*}\right)+\lambda_{n}\left\langle\mathrm{~J}_{\mathrm{E}_{2}}^{\mathrm{p}} \nu_{n}, A x^{*}-A x_{n}\right\rangle+\frac{\mathrm{C}_{\mathrm{q}}\left(\lambda_{n}\|A\|\right)^{\mathrm{q}}}{\mathrm{q}}\left\|\mathrm{J}_{\mathrm{E}_{2}}^{\mathrm{p}} \nu_{n}\right\|^{\mathrm{q}} \\
& \leqslant \Delta_{p}\left(x_{n}, x^{*}\right)-\left(\lambda_{n}-\frac{C_{q}\left(\lambda_{n}\|A\|\right)^{q}}{q}\right)\left\|v_{n}\right\|^{p} \text {. }
\end{aligned}
$$

By the assumption of $\left\{\lambda_{n}\right\}$, we have

$$
\Delta_{\mathfrak{p}}\left(y_{n}, x^{*}\right) \leqslant \Delta_{p}\left(x_{n}, x^{*}\right),
$$

so that $\Gamma \subset D_{n+1}$ for all $n \geqslant 1$. Next, we show $\Gamma \subset C_{n+1}$. Note that $\Gamma \subset C_{1}=C$. Suppose $\Gamma \subset C_{n}$ for $n \geqslant 1$, then for all $x^{*} \in \Gamma \subset C_{n}$, from (2.6), (3.1), (3.2) and (3.6), we obtain

$$
\begin{aligned}
& \Delta_{\mathfrak{p}}\left(z_{n}, x^{*}\right)=\Delta_{\mathrm{p}}\left(\mathrm{J}_{\mathrm{E}_{1}^{*}}^{\mathrm{q}}\left(\alpha_{\mathrm{n}} \mathrm{J}_{\mathrm{E}_{1}}^{\mathrm{p}} y_{\mathrm{n}}+\left(1-\alpha_{\mathrm{n}}\right) \mathrm{J}_{\mathrm{E}_{1}}^{\mathrm{p}} \mathrm{T} x_{\mathrm{n}}\right), x^{*}\right) \\
& \leqslant \alpha_{n} \Delta_{p}\left(y_{n}, x^{*}\right)+\left(1-\alpha_{n}\right) \Delta_{p}\left(T x_{n}, x^{*}\right) \\
& \leqslant \alpha_{n} \Delta_{p}\left(y_{n}, x^{*}\right)+\left(1-\alpha_{n}\right)\left(\Delta_{p}\left(x_{n}, x^{*}\right)+k \Delta_{p}\left(T x_{n}, x_{n}\right)\right) \\
& \leqslant \Delta_{\mathfrak{p}}\left(x_{n}, x^{*}\right)+k \Delta_{p}\left(T x_{n}, x_{n}\right) \\
& \leqslant \Delta_{p}\left(x_{n}, x^{*}\right)+\frac{\kappa}{1-\kappa}\left\langle x^{*}-x_{n}, J_{E_{1}}^{p} T x_{n}-J_{E_{1}}^{p} x_{n}\right\rangle \text {. }
\end{aligned}
$$

From (2.1), we get

$$
\Delta_{\mathfrak{p}}\left(z_{n}, x^{*}\right)=\Delta_{\mathfrak{p}}\left(z_{n}, x_{n}\right)+\Delta_{\mathfrak{p}}\left(x_{n}, x^{*}\right)+\left\langle x_{n}-x^{*}, \mathrm{~J}_{\mathrm{E}_{1}}^{\mathrm{p}} z_{\mathfrak{n}}-\mathrm{J}_{\mathrm{E}_{1}}^{\mathrm{p}} x_{\mathfrak{n}}\right\rangle
$$

By (3.7) and (3.8), we obtain

$$
\Delta_{\mathrm{p}}\left(z_{n}, x_{n}\right) \leqslant \frac{\kappa}{1-\kappa}\left\langle x^{*}-x_{n}, \mathrm{~J}_{\mathrm{E}_{1}}^{\mathrm{p}} \mathrm{T} x_{n}-\mathrm{J}_{\mathrm{E}_{1}}^{\mathrm{p}} x_{n}\right\rangle+\left\langle x^{*}-x_{n}, \mathrm{~J}_{\mathrm{E}_{1}}^{\mathrm{p}} z_{n}-\mathrm{J}_{\mathrm{E}_{1}}^{\mathrm{p}} x_{n}\right\rangle .
$$

This shows that $x^{*} \in C_{n+1}$, which implies $\Gamma \subset C_{n+1}$ for all $n \geqslant 1$. Thus, $D_{n+1} \cap C_{n+1}$ is nonempty. So $\left\{x_{n}\right\}$ is well-defined.

From (3.1) and (2.4), we have

$$
\left\langle\mathrm{J}_{\mathrm{E}_{1}}^{\mathrm{p}} \mathrm{x}_{0}-\mathrm{J}_{\mathrm{E}_{1}}^{\mathrm{p}} \mathrm{x}_{\mathrm{n}}, z-\mathrm{x}_{\mathrm{n}}\right\rangle \leqslant 0, \quad z \in \mathrm{C}_{n} .
$$

Since $\Gamma \subset C_{n}$, we have

$$
\left\langle\mathrm{J}_{\mathrm{E}_{1}}^{\mathrm{p}} \mathrm{x}_{0}-\mathrm{J}_{\mathrm{E}_{1}}^{\mathrm{p}} \mathrm{x}_{\mathrm{n}}, x^{*}-\mathrm{x}_{\mathrm{n}}\right\rangle \leqslant 0, \quad x^{*} \in \Gamma .
$$

By (2.5) and for all $x^{*} \in \Gamma$, we have

$$
\begin{aligned}
\Delta_{\mathfrak{p}}\left(\mathrm{x}_{\mathfrak{n}}, \mathrm{x}_{0}\right) & \leqslant \Delta_{\mathfrak{p}}\left(\mathrm{x}^{*}, \mathrm{x}_{0}\right)-\Delta_{\mathfrak{p}}\left(\mathrm{x}^{*}, \mathrm{x}_{\mathfrak{n}}\right) \\
& \leqslant \Delta_{\mathfrak{p}}\left(\mathrm{x}^{*}, \mathrm{x}_{0}\right) .
\end{aligned}
$$


This shows that $\left\{\Delta_{\mathfrak{p}}\left(x_{n}, x_{0}\right)\right\}$ is bounded. Hence, $\left\{x_{n}\right\}$ is bounded. By the construction of $C_{n}$, we get $x_{m} \in C_{m} \subset C_{n}$ and $x_{n}=\Pi_{C_{n}} x_{0}$, for any $m \geqslant n$. From (2.5), we obtain

$$
\begin{aligned}
\Delta_{\mathfrak{p}}\left(x_{\mathfrak{m}}, x_{\mathfrak{n}}\right) & =\Delta_{\mathfrak{p}}\left(x_{m}, \Pi_{C_{n}} x_{0}\right) \leqslant \Delta_{\mathfrak{p}}\left(x_{m}, x_{0}\right)-\Delta_{\mathfrak{p}}\left(\Pi_{C_{n}} x_{0}, x_{0}\right) \\
& =\Delta_{\mathfrak{p}}\left(x_{m}, x_{0}\right)-\Delta_{\mathfrak{p}}\left(x_{n}, x_{0}\right) .
\end{aligned}
$$

Since $x_{n}=\Pi_{C_{n}} x_{0}$ and $x_{m}=\Pi_{C_{m}} x_{0} \in C_{m} \subset C_{n}$, we have $\Delta_{p}\left(x_{n}, x_{0}\right) \leqslant \Delta_{p}\left(x_{m}, x_{0}\right)$ for all $m \geqslant n$. This implies that $\left\{\Delta_{\mathfrak{p}}\left(x_{n}, x_{0}\right)\right\}$ is nondecreasing and hence the $\operatorname{limit}_{\lim } \rightarrow \infty \Delta_{\mathfrak{p}}\left(x_{n}, x_{0}\right)$ exists. From (3.10) we obtain $\Delta_{p}\left(x_{n}, x_{m}\right) \rightarrow 0$ as $m, n \rightarrow \infty$. From (2.2) we have $\left\|x_{n}-x_{m}\right\| \rightarrow 0$ as $m, n \rightarrow \infty$. Hence, $\left\{x_{n}\right\}$ is a Cauchy sequence in $C \subset E_{1}$, so there exists $x \in E_{1}$ such that $x_{n} \rightarrow x$ as $n \rightarrow \infty$.

By using (2.1) and (2.4), we have

$$
\begin{aligned}
& \Delta_{\mathfrak{p}}\left(\mathrm{x}_{0}, \Pi_{\Gamma} \mathrm{x}_{0}\right) \geqslant \Delta_{\mathfrak{p}}\left(\mathrm{x}_{0}, \mathrm{x}_{\mathrm{n}+1}\right) \\
& =\Delta_{p}\left(x_{0}, x_{n}\right)+\Delta_{p}\left(x_{n}, x_{n+1}\right)+\left\langle x_{n}-x_{n+1}, J_{E_{1}}^{p} x_{0}-J_{E_{1}}^{p} x_{n}\right\rangle \\
& \geqslant \Delta_{\mathfrak{p}}\left(x_{0}, x_{\mathfrak{n}}\right)+\Delta_{\mathfrak{p}}\left(x_{n}, x_{n+1}\right) \\
& \geqslant \Delta_{\mathfrak{p}}\left(x_{0}, x_{n-1}\right)+\Delta_{\mathfrak{p}}\left(x_{n-1}, x_{\mathfrak{n}}\right)+\Delta_{\mathfrak{p}}\left(x_{\mathfrak{n}}, x_{\mathfrak{n}+1}\right) \\
& \text { : } \\
& \geqslant \sum_{i=0}^{n} \Delta_{p}\left(x_{i}, x_{i+1}\right)
\end{aligned}
$$

Consequently, $\sum_{i=0}^{\infty} \Delta_{p}\left(x_{i}, x_{i+1}\right)<\infty$, which from (2.2) yields $\sum_{i=0}^{\infty}\left\|x_{n}-x_{n+1}\right\|^{p}<\infty$. This implies that

$$
\lim _{n \rightarrow \infty}\left\|x_{n}-x_{n+1}\right\|=0
$$

Since $x_{n+1}=\Pi_{C_{n+1}} x_{0} \in C_{n+1}$, we have,

$$
\Delta_{p}\left(z_{n}, x_{n}\right) \leqslant \frac{\kappa}{1-\kappa}\left\langle x_{n+1}-x_{n}, J_{E_{1}}^{p} T x_{n}-J_{E_{1}}^{p} x_{n}\right\rangle+\left\langle x_{n+1}-x_{n}, J_{E_{1}}^{p} z_{n}-J_{E_{1}}^{p} x_{n}\right\rangle .
$$

From (3.11) and (2.2), we obtain

$$
\lim _{n \rightarrow \infty}\left\|x_{n}-z_{n}\right\|=0
$$

Since $x_{n+1}=\Pi_{D_{n+1}} x_{0} \in D_{n+1}$, we get

$$
\Delta_{p}\left(y_{n}, x_{n+1}\right) \leqslant \Delta_{p}\left(x_{n}, x_{n+1}\right) .
$$

From (3.11), we have

$$
\lim _{n \rightarrow \infty}\left\|y_{n}-x_{n+1}\right\|=0
$$

so,

$$
\lim _{n \rightarrow \infty}\left\|x_{n}-y_{n}\right\|=0 \text {. }
$$

Since $\mathrm{J}_{\mathrm{E}_{1}}^{\mathrm{p}}$ is norm-to-norm uniformly continuous, from (3.1), we get

$$
\begin{aligned}
\left\|\mathrm{J}_{1} z_{n}-\mathrm{JE}_{1} x_{n}\right\| & =\left\|\alpha_{n}\left(\mathrm{JE}_{1}^{\mathrm{p}} y_{n}-\mathrm{JE}_{\mathrm{E}_{1}}^{\mathrm{p}} x_{n}\right)+\left(1-\alpha_{n}\right)\left(\mathrm{JE}_{1}^{\mathrm{p}} \mathrm{T} x_{n}-\mathrm{J}_{\mathrm{E}_{1}}^{\mathrm{p}} x_{n}\right)\right\| \\
& \geqslant\left(1-\alpha_{n}\right)\left\|\mathrm{J}_{\mathrm{E}_{1}}^{\mathrm{p}} \mathrm{T} x_{n}-\mathrm{J}_{\mathrm{E}_{1}}^{\mathrm{p}} x_{n}\right\|-\alpha_{n}\left\|\mathrm{~J}_{\mathrm{E}_{1}}^{\mathrm{p}} y_{n}-\mathrm{J}_{\mathrm{E}_{1}}^{\mathrm{p}} x_{n}\right\| .
\end{aligned}
$$

This implies that

$$
\left(1-\alpha_{n}\right)\left\|J_{\mathrm{E}_{1}}^{\mathrm{p}} \mathrm{T} x_{n}-\mathrm{J}_{\mathrm{E}_{1}}^{\mathrm{p}} x_{n}\right\| \leqslant \alpha_{n}\left\|\mathrm{~J}_{\mathrm{E}_{1}}^{\mathrm{p}} y_{n}-\mathrm{J}_{\mathrm{E}_{1}}^{\mathrm{p}} x_{n}\right\|+\left\|\mathrm{J}_{\mathrm{E}_{1}}^{\mathrm{p}} z_{n}-\mathrm{J}_{\mathrm{E}_{1}}^{\mathrm{p}} x_{n}\right\| .
$$


Since $\mathrm{J}_{\mathrm{E}_{1}^{*}}^{\mathrm{q}}$ is norm-to-norm uniformly continuous, by setting $\mathrm{n} \rightarrow \infty$ in (3.14), from (3.12), (3.13) and $\left\{\alpha_{n}\right\} \subset[c, d] \subset(0,1)$, we have

$$
\lim _{n \rightarrow \infty}\left\|T x_{n}-x_{n}\right\|=0 .
$$

By the closedness of $T$, from $x_{n} \rightarrow x$, we obtain

$$
\mathrm{T} x=x
$$

From (2.2) and (2.4), we have

$$
\begin{aligned}
\Delta_{\mathfrak{p}}\left(x, \Pi_{C} x\right) \leqslant & \left\langle x-\Pi_{C}, J_{\mathrm{E}_{1}}^{\mathrm{p}} x-J_{\mathrm{E}_{1}}^{\mathrm{p}} \Pi_{C} x\right\rangle \\
= & \left\langle x-x_{n}, J_{\mathrm{E}_{1}}^{\mathrm{p}} x-J_{\mathrm{E}_{1}}^{\mathrm{p}} \Pi_{C} x\right\rangle+\left\langle x_{n}-y_{n}, J_{\mathrm{E}_{1}}^{\mathrm{p}} x-J_{\mathrm{E}_{1}}^{\mathrm{p}} \Pi_{C} x\right\rangle \\
& +\left\langle y_{n}-\Pi_{C} x, J_{\mathrm{E}_{1}}^{p} x-J_{\mathrm{E}_{1}}^{\mathrm{p}} \Pi_{C} x\right\rangle \\
\leqslant & \left\langle x-x_{n}, J_{\mathrm{E}_{1}}^{\mathrm{p}} x-J_{\mathrm{E}_{1}}^{\mathrm{p}} \Pi_{C} x\right\rangle+\left\langle x_{n}-y_{n}, J_{\mathrm{E}_{1}}^{\mathrm{p}} x-J_{\mathrm{E}_{1}}^{\mathrm{p}} \Pi_{C} x\right\rangle .
\end{aligned}
$$

Setting $n \rightarrow \infty$ yields $\Delta_{p}\left(x, \Pi_{C} x\right)=0$, we get $x \in C$.

From (3.5), we have

$$
\left(\lambda_{n}-\frac{C_{q}\left(\lambda_{n}\|A\|\right)^{q}}{q}\right)\left\|v_{n}\right\|^{p} \leqslant \Delta_{p}\left(x_{n}, x^{*}\right)-\Delta_{p}\left(y_{n}, x^{*}\right)
$$

This together with $v_{n}=A x_{n}-P_{Q} A x_{n}$ and (3.13) implies that

$$
\lim _{n \rightarrow \infty}\left\|A x_{n}-P_{Q} A x_{n}\right\|=0 .
$$

By (2.3), we have

$$
\begin{aligned}
\left\|A x-P_{Q} A x\right\|^{p}= & \left\langle J_{E_{2}}^{p}\left(A x-P_{Q} A x\right), A x-P_{Q} A x\right\rangle \\
= & \left\langle J_{E_{2}}^{p}\left(A x-P_{Q} A x\right), A x-A x_{n}\right\rangle+\left\langle J_{E_{2}}^{p}\left(A x-P_{Q} A x\right), A x_{n}-P_{Q} A x_{n}\right\rangle \\
& +\left\langle J_{E_{2}}^{p}\left(A x-P_{Q} A x\right), P_{Q} A x_{n}-P_{Q} A x\right\rangle \\
\leqslant & \left\langle J_{E_{2}}^{p}\left(A x-P_{Q} A x\right), A x-A x_{n}\right\rangle+\left\langle J E_{E_{2}}^{p}\left(A x-P_{Q} A x\right), A x_{n}-P_{Q} A x_{n}\right\rangle .
\end{aligned}
$$

From (3.15) and $A x_{n} \rightarrow A x$ as $n \rightarrow \infty$, setting $n \rightarrow \infty$ yields $\left\|A x-P_{Q} A x\right\|^{p}=0$, we have $A x \in Q$. Thus, we conclude that $x_{n} \rightarrow x \in \Gamma$.

By setting $n \rightarrow \infty$ in (3.9), we obtain

$$
\left\langle\mathrm{J}_{\mathrm{E}_{1}}^{\mathrm{p}} \mathrm{x}_{0}-\mathrm{J}_{\mathrm{E}_{1}}^{\mathrm{p}} x, x^{*}-x\right\rangle \leqslant 0, \quad x^{*} \in \Gamma .
$$

By (2.4), we have $x=\Pi_{\Gamma} x_{0}$.

Remark 3.2. Compared with the known results in the literature, our result is very different from those in the following aspects.

- The corresponding iterative algorithms in [16, Theorem 3.2], [18, Theorem 3.1], [19, Theorem 3.1] are extended for developing our algorithm which couples modified CQ method with Nakajo's iteration involved in Bregman quasi-strictly pseudo-contractive mapping in Theorem 3.1. Our iterative scheme in Theorem 3.1 can be viewed as a merger between corresponding iterative algorithms in [16, Theorem 3.2], [18, Theorem 3.1], [19, Theorem 3.1]. 
- The construction of sets such as $\mathrm{C}_{n+1}=\left\{w \in \mathrm{C}_{n}: \Delta_{\mathrm{p}}\left(z_{n}, x_{n}\right) \leqslant \frac{\kappa}{1-\kappa}\left\langle w-x_{n}, \mathrm{~J}_{\mathrm{E}_{1}}^{\mathrm{p}} \mathrm{T} x_{n}-\mathrm{J}_{\mathrm{E}_{1}}^{\mathrm{p}} x_{n}\right\rangle+\right.$ $\left.\left\langle w-x_{n}, J_{\mathrm{E}_{1}}^{\mathrm{p}} z_{n}-\mathrm{J}_{\mathrm{E}_{1}}^{\mathrm{p}} x_{n}\right\rangle\right\}$ in our iterative scheme is very different from the iterative algorithm in [16, Theorem 3.2], because our construction is mainly based on the definition of Bregman quasistrictly pseudo-contractive mapping. Moreover, we attain strong convergence result in a broader framework, the p-uniformly convex and uniformly smooth Banach spaces.

- The technique of proving strong convergence in Theorem 3.1 is different from those in [16, Theorem 3.2], [19, Theorem 3.1], because our technique depends on Lemma 2.1 in Banach spaces.

- The problem of finding a common element of the set of solutions of split feasibility problem and the set of fixed points of a Bregman quasi-strictly pseudo-contractive mapping in our Theorem 3.1 is more general than the problem of finding a solution of split feasibility problem in [16, Theorem 3.2] and the problem of finding an element of the set of fixed points of a Bregman quasi-strictly pseudo-contractive mapping in [19, Theorem 3.1].

Since the class of Bregman quasi-nonexpansive mappings is Bregman quasi-strict pseudo-contractive, the following corollary is obtained by using Theorem 3.1.

Corollary 3.3. Let $\mathrm{E}_{1}$ and $\mathrm{E}_{2}$ be two p-uniformly convex real Banach spaces which are also uniformly smooth. Let $C$ and $Q$ be nonempty closed convex sets of $\mathrm{E}_{1}$ and $\mathrm{E}_{2}$, respectively. Let $\mathrm{A}: \mathrm{E}_{1} \rightarrow \mathrm{E}_{2}$ be a bounded linear operator with its adjoint $\mathrm{A}^{*}: \mathrm{E}_{2}^{*} \rightarrow \mathrm{E}_{1}^{*}$. Let $\mathrm{T}$ be a closed Bregman quasi-nonexpansive mapping from $\mathrm{C}$ to itself. Let sequence $\left\{x_{n}\right\}$ be iteratively generated by $x_{1}=x_{0} \in C, D_{1}=C_{1}=C$,

$$
\left\{\begin{array}{l}
x_{1} \in C, \\
y_{n}=\Pi_{C} J_{E_{1}^{*}}^{q}\left(J_{E_{1}}^{p} x_{n}-\lambda_{n} A^{*} J_{E_{2}}^{p}\left(A x_{n}-P_{Q} A x_{n}\right)\right), \\
z_{n}=J_{E_{1}^{*}}^{q}\left(\alpha_{n} J_{E_{1}}^{p} y_{n}+\left(1-\alpha_{n}\right) J_{E_{1}}^{p} T x_{n}\right), \\
D_{n+1}=\left\{w \in D_{n}: \Delta_{p}\left(y_{n}, w\right) \leqslant \Delta_{p}\left(x_{n}, w\right)\right\}, \\
C_{n+1}=\left\{w \in C_{n}: \Delta_{p}\left(z_{n}, x_{n}\right) \leqslant\left\langle w-x_{n}, J_{E_{1}}^{p} z_{n}-J_{E_{1}}^{p} x_{n}\right\rangle\right\}, \\
x_{n+1}=\Pi_{D_{n+1} \cap C_{n+1} x_{0}, \quad n \geqslant 1 .}
\end{array}\right.
$$

Assume that $\left\{\alpha_{n}\right\} \subset[c, d] \subset(0,1)$ and $\left\{\lambda_{n}\right\} \subset[a, b] \subset\left(0,\left(\frac{q}{c_{q}\|A\|^{q}}\right)^{\frac{1}{q-1}}\right)$. Then the sequence $\left\{x_{n}\right\}$ defined by (3.16) converges strongly to $\Pi_{\Gamma} x_{0}$.

Typical examples of both uniformly convex and uniformly smooth Banach spaces are $\mathrm{L}^{\mathrm{p}}$, where $\mathrm{p}>1$. Then we have the following corollary.

Corollary 3.4. Let $\mathrm{E}_{1}$ and $\mathrm{E}_{2}$ be two $\mathrm{L}^{\mathrm{p}}$ spaces with $2 \leqslant \mathrm{p}<\infty, \mathrm{C} \subset \mathrm{E}_{1}$ and $\mathrm{Q} \subset \mathrm{E}_{2}$ be two nonempty closed convex sets. Let $A: E_{1} \rightarrow E_{2}$ be a bounded linear operator with its adjoint $A^{*}: E_{2}^{*} \rightarrow E_{1}^{*}$. Let $T$ be a closed Bregman quasi-strictly pseudo-contractive mapping from $\mathrm{C}$ to itself. Let sequence $\left\{x_{n}\right\}$ be iteratively generated by $\mathrm{x}_{1}=\mathrm{x}_{0} \in \mathrm{C}, \mathrm{D}_{1}=\mathrm{C}_{1}=\mathrm{C}$,

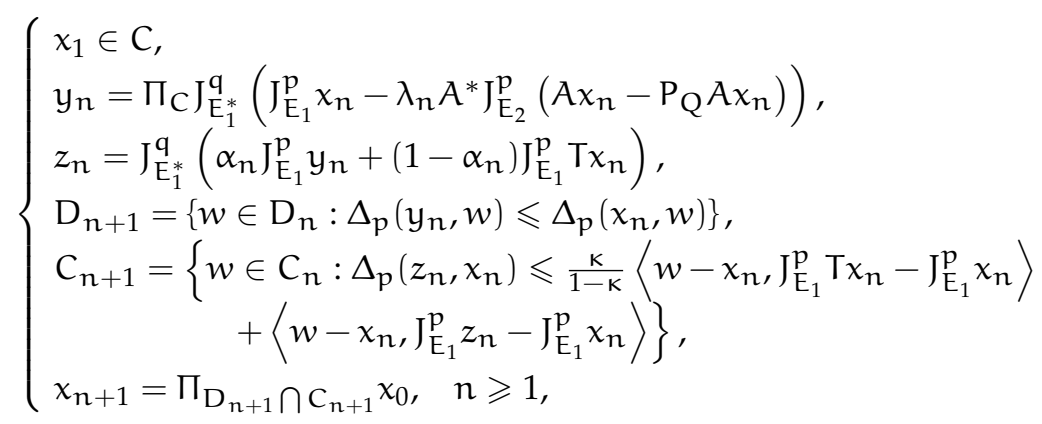

where $\mathrm{k} \in[0,1)$. Assume that $\left\{\alpha_{\mathrm{n}}\right\} \subset[\mathrm{c}, \mathrm{d}] \subset(0,1)$ and $\left\{\lambda_{\mathrm{n}}\right\} \subset[\mathrm{a}, \mathrm{b}] \subset\left(0,\left(\frac{\mathrm{q}}{\mathrm{C}_{\mathrm{q}}\|\mathrm{A}\|^{\mathrm{q}}}\right)^{\frac{1}{\mathrm{q}-1}}\right)$. Then the sequence $\left\{x_{n}\right\}$ defined by (3.17) converges strongly to $\Pi_{\Gamma} x_{0}$. 
Finally, we consider the following special case in Hilbert spaces.

Corollary 3.5. Let $\mathrm{H}_{1}$ and $\mathrm{H}_{2}$ be two real Hilbert spaces and $\mathrm{C} \subset \mathrm{H}_{1}$ and $\mathrm{Q} \subset \mathrm{H}_{2}$ be two nonempty closed convex sets. Let $\mathrm{A}: \mathrm{H}_{1} \rightarrow \mathrm{H}_{2}$ be a bounded linear operator with its adjoint $\mathrm{A}^{*}$. Let $\mathrm{T}$ be a closed quasi-strictly pseudo-contractive mapping from $\mathrm{C}$ to itself. Let the sequence $\left\{x_{n}\right\}$ be iteratively generated by $x_{1}=x_{0} \in C$, $\mathrm{D}_{1}=\mathrm{C}_{1}=\mathrm{C}$,

$$
\left\{\begin{array}{l}
x_{1} \in C \\
y_{n}=P_{C}\left(x_{n}-\lambda_{n} A^{*}\left(A x_{n}-P_{Q} A x_{n}\right)\right) \\
z_{n}=\alpha_{n} y_{n}+\left(1-\alpha_{n}\right) T x_{n} \\
D_{n+1}=\left\{w \in D_{n}:\left\|y_{n}-w\right\| \leqslant\left\|x_{n}-w\right\|\right\} \\
C_{n+1}=\left\{w \in C_{n}:\left\|z_{n}-x_{n}\right\|^{2} \leqslant \frac{2 k}{1-k}\left\langle w-x_{n}, T x_{n}-x_{n}\right\rangle+2\left\langle w-x_{n}, z_{n}-x_{n}\right\rangle\right\} \\
x_{n+1}=P_{D_{n+1} \cap C_{n+1}} x_{0}, \quad n \geqslant 1,
\end{array}\right.
$$

where $\mathrm{k} \in[0,1)$. Assume that $\left\{\alpha_{n}\right\} \subset[\mathrm{c}, \mathrm{d}] \subset(0,1)$ and $\left\{\lambda_{\mathrm{n}}\right\} \subset[\mathrm{a}, \mathrm{b}] \subset\left(0, \frac{2}{\|\mathrm{~A}\|^{2}}\right)$. Then the sequence $\left\{\mathrm{x}_{\mathrm{n}}\right\}$ defined by (3.18) converges strongly to $\mathrm{P}_{\Gamma} \mathrm{x}_{0}$.

\section{Numerical example}

In this section, in order to demonstrate the effectiveness, realization and convergence of algorithm of Theorem 3.1, we consider the following simple example in $\left(\mathbb{R},\|\cdot\|_{2}\right)$. All codes were written in Matlab R2014a and run on Lenovo i-5 Dual-Core laptop.

Example 4.1 (Numerical Example). Let $E_{1}=E_{2}=\mathbb{R}$ with the standard norm $|\cdot|=\|\cdot\|$ for all $x, y \in \mathbb{R}$. Let $C=[0,+\infty)$, and $Q=(-\infty, 0]$. In (3.1), we take $\alpha_{n}=6 / 7, k=1 / 9, \lambda_{n}=2, T x=2 x$, for all $x \in C$ and $A x=1 / 2 x$, for all $x \in \mathbb{R}$.

Then the scheme (3.1) can be simplified as

$$
\left\{\begin{array}{l}
x_{1} \in C, \\
y_{n}=P_{C}\left(x_{n}-\left(I-P_{Q}\right) \frac{1}{2} x_{n}\right), \\
z_{n}=\frac{6}{7} y_{n}+\frac{2}{7} x_{n}, \\
D_{n+1}=\left\{w \in D_{n}: w \leqslant \frac{x_{n}^{2}-y_{n}^{2}}{2 x_{n}-2 y_{n}}\right\}, \\
C_{n+1}=\left\{w \in C_{n}: w \leqslant \frac{3 x_{n}^{2}-4 z_{n}^{2}}{7 x_{n}-8 z_{n}}\right\}, \\
x_{n+1}=P_{D_{n+1} \cap C_{n+1}} x_{1}, \quad n \geqslant 1 .
\end{array}\right.
$$

Choosing initial values $x_{1}=6$ and $x_{1}=3$, respectively, we see that figure and numerical results demonstrate Theorem 3.1. Table 1 and Figure 1 show that the sequence $\left\{x_{n}\right\}$ generated by the above algorithm converges to 0 .

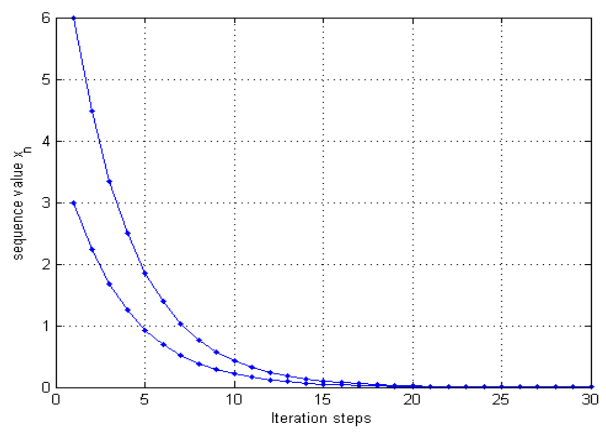

Figure 1: The convergence of $x_{n}$ with initial values 6 and 3, respectively. 
Table 1: The values of the sequence $x_{n}$

\begin{tabular}{|l|c|c|}
\hline $\mathrm{n}$ & $x_{\mathrm{n}}$ & $x_{\mathrm{n}}$ \\
\hline 1 & 6.000000000000000 & 3.000000000000000 \\
\hline 2 & 4.476190476190476 & 2.238095238095238 \\
\hline 3 & 3.339380196523054 & 1.669690098261527 \\
\hline 4 & 2.491283638675928 & 1.245641819337964 \\
\hline 5 & 1.858576682821724 & 0.929288341410862 \\
\hline$\ldots$ & $\ldots$ & $\ldots$ \\
\hline 27 & 0.002950130794513 & 0.001475065397256 \\
\hline 28 & 0.002200891227652 & 0.001100445613826 \\
\hline 29 & 0.001641934725392 & 0.000820967362696 \\
\hline 30 & 0.001224935430054 & 0.000612467715027 \\
\hline
\end{tabular}

\section{Acknowledgment}

This work was supported by the Innovation Program of Shanghai Municipal Education Commission (15ZZ068), Ph.D. Program Foundation of Ministry of Education of China (20123127110002) and Program for Shanghai Outstanding Academic Leaders in Shanghai City (15XD1503100).

\section{References}

[1] R. P. Agarwal, J.-W. Chen, Y. J. Cho, Strong convergence theorems for equilibrium problems and weak Bregman relatively nonexpansive mappings in Banach spaces, J. Inequal. Appl., 2013 (2013), 16 pages. 1

[2] Y. I. Alber, Metric and generalized projection operators in Banach spaces: properties and applications, Theory and applications of nonlinear operators of accretive and monotone type, Lecture Notes in Pure and Appl. Math., Dekker, New York, 178 (1996), 15-50. 2, 2

[3] H. H. Bauschke, J. M. Borwein, P. L. Combettes, Essential smoothness, essential strict convexity, and Legendre functions in Banach spaces, Commun. Contemp. Math., 3 (2001), 615-647. 1

[4] L. M. Brégman, A relaxation method of finding a common point of convex sets and its application to the solution of problems in convex programming, (Russian) Ž. Vyčisl. Mat. i Mat. Fiz., 7 (1967), 620-631. 1

[5] C. Byrne, Iterative oblique projection onto convex sets and the split feasibility problem, Inverse Problems, 18 (2002), 441-453. 1

[6] C. Byrne, A unified treatment of some iterative algorithms in signal processing and image reconstruction, Inverse Problems, 20 (2004), 103-120. 1

[7] L.-C. Ceng, Q. H. Ansari, J.-C. Yao, An extragradient method for solving split feasibility and fixed point problems, Comput. Math. Appl., 64 (2012), 633-642. 1

[8] L.-C. Ceng, Q. H. Ansari, J.-C. Yao, Relaxed extragradient methods for finding minimum-norm solutions of the split feasibility problem, Nonlinear Anal., 75 (2012), 2116-2125.

[9] L.-C. Ceng, C.-M. Chen, C.-F. Wen, Generalized mixed equilibria, variational inequalities and common fixed point problems, J. Nonlinear Convex Anal., 16 (2015), 2365-2400.

[10] L.-C. Ceng, C.-F. Wen, Implicit hybrid steepest-descent methods for generalized mixed equilibria with variational inclusions and variational inequalities, J. Nonlinear Convex Anal., 17 (2016), 987-1012.

[11] L.-C. Ceng, M.-M. Wong, A. Petruşel, J.-C. Yao, Relaxed implicit extragradient-like methods for finding minimum-norm solutions of the split feasibility problem, Fixed Point Theory, 14 (2013), 327-344. 1

[12] Y. Censor, T. Bortfeld, B. Martin, A. Trofimov, A unified approach for inversion problems in intensity-modulated radiation therapy, Phys. Med. Biol., 51 (2006), 2353-2365. 1

[13] Y. Censor, T. Elfving, A multiprojection algorithm using Bregman projections in a product space, Numer. Algorithms, 8 (1994), 221-239. 1

[14] Y. Censor, T. Elfving, N. Kopf, T. Bortfeld, The multiple-sets split feasibility problem and its applications for inverse problems, Inverse Problems, 21 (2005), 2071-2084. 1

[15] Y. Censor, A. Motova, A. Segal, Perturbed projections and subgradient projections for the multiple-sets split feasibility problem, J. Math. Anal. Appl., 327 (2007), 1244-1256. 1

[16] S. Y. Cho, S. M. Kang, Approximation of viscosity zero points of accretive operators in a Banach space, Filomat, 28 (2014), 2175-2184. 1, 3.2

[17] N.-N. Fang, Y.-P. Gong, Viscosity iterative methods for split variational inclusion problems and fixed point problems of a nonexpansive mapping, Commun. Optim. Theory, 2016 (2016), 15 pages. 1 
[18] K. Nakajo, W. Takahashi, Strong convergence theorems for nonexpansive mappings and nonexpansive semigroups, J. Math. Anal. Appl., 279 (2003), 372-379. 1, 3.2

[19] X.-L. Qin, S. Y. Cho, L. Wang, Algorithms for treating equilibrium and fixed point problems, Fixed Point Theory Appl., 2013 (2013), 15 pages. 1, 3.2

[20] X.-L. Qin, J.-C. Yao, Weak convergence of a Mann-like algorithm for nonexpansive and accretive operators, J. Inequal. Appl., 2016 (2016), 9 pages. 1

[21] F. Schöpfer, T. Schuster, A. K. Louis, An iterative regularization method for the solution of the split feasibility problem in Banach spaces, Inverse Problems, 24 (2008), 20 pages. 1, 1

[22] W. Takahashi, The split feasibility problem in Banach spaces, J. Nonlinear Convex Anal., 15 (2014), 1349-1355. 1

[23] G. C. Ugwunnadi, B. Ali, I. Idris, S. M. Minjibir, Strong convergence theorem for quasi-Bregman strictly pseudocontractive mappings and equilibrium problems in Banach spaces, Fixed Point Theory Appl., 2014 (2014), 16 pages. 1, 2, 2.6

[24] F.-H. Wang, A new algorithm for solving the multiple-sets split feasibility problem in Banach spaces, Numer. Funct. Anal. Optim., 35 (2014), 99-110. 1, 2.1

[25] Z.-M. Wang, Strong convergence theorems for Bregman quasi-strict pseudo-contractions in reflexive Banach spaces with applications, Fixed Point Theory Appl., 2015 (2015), 17 pages. 1, 2.5

[26] Y.-H. Yao, G. Marino, L. Muglia, A modified Korpelevich's method convergent to the minimum-norm solution of a variational inequality, Optimization, 63 (2014), 559-569. 1

[27] Y.-H. Yao, G. Marino, H.-K. Xu, Y.-C. Liou, Construction of minimum-norm fixed points of pseudocontractions in Hilbert spaces, J. Inequal. Appl., 2014 (2014), 14 pages.

[28] Y.-H. Yao, M. Postolache, S. M. Kang, Strong convergence of approximated iterations for asymptotically pseudocontractive mappings, Fixed Point Theory Appl., 2014 (2014), 13 pages. 1 